\title{
Interfacial slip friction at a fluid-solid cylindrical boundary
}

Kannam, S.; Todd, Billy; Hansen, Jesper Schmidt; Daivis, Peter

Published in:

Journal of Chemical Physics

DOI:

$10.1063 / 1.4730167$

Publication date:

2012

Document Version

Publisher's PDF, also known as Version of record

Citation for published version (APA):

Kannam, S., Todd, B., Hansen, J. S., \& Daivis, P. (2012). Interfacial slip friction at a fluid-solid cylindrical boundary. Journal of Chemical Physics, 136(24). https://doi.org/10.1063/1.4730167

\section{General rights}

Copyright and moral rights for the publications made accessible in the public portal are retained by the authors and/or other copyright owners and it is a condition of accessing publications that users recognise and abide by the legal requirements associated with these rights.

- Users may download and print one copy of any publication from the public portal for the purpose of private study or research.

- You may not further distribute the material or use it for any profit-making activity or commercial gain.

- You may freely distribute the URL identifying the publication in the public portal.

Take down policy

If you believe that this document breaches copyright please contact rucforsk@kb.dk providing details, and we will remove access to the work immediately and investigate your claim. 


\title{
Interfacial slip friction at a fluid-solid cylindrical boundary
}

\author{
Sridhar Kumar Kannam, ${ }^{1, a)}$ B. D. Todd, ${ }^{1, b)}$ J. S. Hansen, ${ }^{2, c)}$ and Peter J. Daivis ${ }^{3, d)}$ \\ ${ }^{1}$ Mathematics Discipline, Faculty of Engineering and Industrial Science and Centre for Molecular Simulation, \\ Swinburne University of Technology, Melbourne, Victoria 3122, Australia \\ ${ }^{2}$ DNRF Center 'Glass and Time', IMFUFA, Department of Science, Systems and Models, Roskilde University, \\ DK-4000 Roskilde, Denmark \\ ${ }^{3}$ School of Applied Sciences, RMIT University, Melbourne, Victoria 3001, Australia
}

(Received 16 February 2012; accepted 4 June 2012; published online 27 June 2012)

\begin{abstract}
Recently we proposed a method to calculate the interfacial friction coefficient between fluid and solid at a planar interface. In this work we extend the method to cylindrical systems where the friction coefficient is curvature dependent. We apply the method to methane flow in carbon nanotubes, and find good agreement with non-equilibrium molecular dynamics simulations. The proposed method is robust, general, and can be used to predict the slip for cylindrical nanofluidic systems. () 2012 American Institute of Physics. [http://dx.doi.org/10.1063/1.4730167]
\end{abstract}

\section{INTRODUCTION}

The hydrodynamic boundary condition $(\mathrm{BC})$ is now a subject of greater interest than ever before, even though the problem of formulating the correct $\mathrm{BC}$ has existed from the beginning of the 19th century. Since then, many researchers have attempted to formulate a general $\mathrm{BC}$ at a fluid-solid interface. The 21 st century has seen revolutionary advancement in nanoscience and nanotechnology, which in turn, poses many fundamental questions about the nature of fluid flow in nanometric pores such as carbon nanotubes (CNTs) and aquaporins. Among them, one of the most important is the prediction of fluid slip.

To describe a hydrodynamic problem, the $\mathrm{BC}$ should be specified or known a priori. This BC along with the fluid transport coefficients is used in the Navier-Stokes equations to solve for the relevant flow properties. ${ }^{1,2}$ The fluid transport coefficients are intrinsic to the fluid and there are several methods of finding them accurately. As the BC cannot be derived from Navier-Stokes hydrodynamics, one often assumes the no-slip BC, according to which the tangential velocity of the fluid relative to the adjacent solid is zero irrespective of the nature of both fluid and solid. ${ }^{1,2}$ In principle, the $\mathrm{BC}$ is a property of the fluid-solid combination, but the no-slip BC is widely accepted mainly for two reasons. The first is, even if some slip is present its effect is negligible on a macroscale. ${ }^{3}$ The second is the experimental difficulties in probing and measuring the fluid velocity within sub-nanometer length scales from the solid surface, which is needed for correct formulation of the BC. ${ }^{4}$ Recent developments have enabled the fabrication of nanofluidic devices and measurement of nanolitre flow rates of fluid. ${ }^{5}$ Over the last decade many experimental and simulation studies have found volumetric flow rates enhanced by orders of magnitude compared to the classical hydrodynamics prediction with the

\footnotetext{
a)Electronic mail: urssrisri@gmail.com.

b)Electronic mail: btodd@swin.edu.au.

c)Electronic mail: jschmidt@ruc.dk.

d) Electronic mail: peter.daivis@rmit.edu.au.
}

no-slip BC in carbon nanopores. The observed flow enhancement is due to the non-zero fluid velocity (velocity slip) at the solid, and hence the no-slip BC is no longer valid. However, the enhancement data are widely scattered ${ }^{6-17}$ hence, the formulation of a precise $\mathrm{BC}$ is a subject of great interest and significant challenge in nanofluidic research. ${ }^{18-27}$

In the steady state, the fluid shear stress $\sigma_{x y}$ must be continuous across the channel. Navier ${ }^{28}$ proposed the first slip BC by relating this shear stress to the fluid slip velocity $u_{s}$ at the wall via the fluid-solid interfacial friction coefficient $\xi_{0}$,

$$
\sigma_{x y}=\xi_{0} u_{s}
$$

where $x$ and $y$ are shearing and confining directions, respectively. Using the above relation with Newton's law of viscosity, which relates the shear stress with the strain rate $\dot{\gamma}$ (at the wall) via the fluid viscosity $\eta_{0}$,

$$
\sigma_{x y}=\eta_{0} \dot{\gamma},
$$

Navier derived the slip BC

$$
u_{s}=\frac{\eta_{0}}{\xi_{0}} \dot{\gamma} .
$$

Here $\frac{\eta_{0}}{\xi_{0}}$ has the units of length and is identified as the slip length $L_{s}$, which is the distance into the wall at which the tangent to the fluid velocity value is zero in a field driven flow. The strain rate of the fluid at the wall can be determined using the Navier-Stokes equations, given the shear viscosity. ${ }^{1,2}$ To use the above $\mathrm{BC}$, one needs to know the interfacial friction between fluid and solid. The hydrodynamic friction between a fluid and solid is not understood as well as the friction between two solid surfaces in contact. Recently, Hansen et $_{\text {al. }}{ }^{24}$ proposed a method to calculate the interfacial friction coefficient between a fluid and solid at a planar boundary. Here we extend the method to a cylindrical boundary where the friction coefficient is curvature dependent. We apply the method to calculate the friction coefficient and hence the slip length for methane flow in CNTs of various diameters and verify that this slip length agrees with the one obtained from nonequilibrium molecular dynamics (NEMD) simulations. 


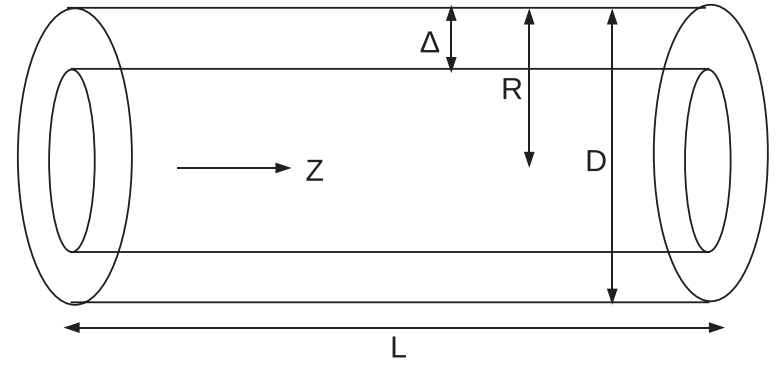

FIG. 1. Schematic representation of the system.

\section{METHODOLOGY}

\section{A. The wall-fluid friction coefficient}

Assume that a fluid is confined in a cylindrical tube of radius $R$ and length $L$. Let $z$ be the fluid streaming direction (axial direction). We consider a cylindrical fluid annulus (or slab) with a constant mass $m$, and average width of one molecular diameter $\Delta$ close to the solid surface, see Fig. 1. In the case of slip flow, this fluid layer slips over the adjacent solid surface. We have developed a method to calculate the interfacial friction between this fluid layer and solid surface, which characterizes the slip between them. ${ }^{24-27}$ Even though this method was developed for planar interfaces, the determination of the friction coefficient is found to be similar for cylindrical interfaces. The only difference is that we consider a cylindrical fluid annulus, whereas for a planar boundary we consider a planar fluid slab near the solid boundary. We refer the reader to our original paper by Hansen et al. ${ }^{24}$ for full details of the method.

\section{B. The wall-fluid slip length}

Here, we derive an explicit expression for slip length using the slab centre of mass $(\mathrm{CM})$ velocity integral boundary condition (IBC) and verify that our slip length is equal to the Navier slip length in the limit as the slab width goes to zero.

The Navier-Stokes equation for fluid flow in a cylindrical tube when acted upon by an external force per unit mass, $F_{e}$, is $^{1,2}$

$$
\frac{1}{r} \frac{\partial}{\partial r}\left(r \frac{\partial u_{z}(r)}{\partial r}\right)=-\frac{\rho F_{e}}{\eta_{0}},
$$

with general solution

$$
u_{z}(r)=-\left(\frac{\rho F_{e}}{4 \eta_{0}}\right) r^{2}+C_{1} \ln (r)+C_{2} .
$$

Since $u_{z}$ is finite at $r=0, C_{1}$ must always be zero. The general IBC is expressed as ${ }^{24}$

$$
\left\langle u_{\text {slab }}\right\rangle=\frac{1}{\Delta} \int_{R-\Delta}^{R} u(r) d r .
$$

Applying this IBC, the solution to the above boundary value problem is

$$
u_{z}(r)=\left(\frac{\rho F_{e}}{12 \eta_{0}}\right)\left(3 R^{2}-3 r^{2}+\Delta^{2}-3 R \Delta\right)+u_{\text {slab }} .
$$

One can obtain the classical solution of a no-slip HagenPoiseuille flow in a cylindrical tube by setting $u_{\text {slab }}=0$, i.e., the slip velocity to be zero.

Let $F_{z}^{\prime}$ be the shearing frictional force on the fluid slab due to the wall and $F_{z}^{\prime \prime}$ is the shearing force between fluid slab and the rest of the fluid adjacent to it, then in the steady state we have

$$
\left\langle F_{z}^{\prime}\right\rangle+\left\langle F_{z}^{\prime \prime}\right\rangle+m F_{e}=0
$$

The wall-slab shear force is proportional to the relative velocity between the wall and slab for sufficiently small velocities

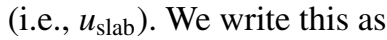

$$
\left\langle F_{x}^{\prime}\right\rangle=-\zeta_{0}\left\langle u_{\text {slab }}\right\rangle
$$

where $\zeta_{0}$ is the zero-frequency friction term and the slab-fluid shearing force is given by Newton's law of viscosity

$$
\left\langle F_{z}^{\prime \prime}\right\rangle=A \eta_{0}\langle\dot{\gamma}\rangle=\left.A \eta_{0} \frac{\partial u}{\partial r}\right|_{r=R-\Delta},
$$

where $A=2 \pi(R-\Delta) L_{z}$ is the surface area of the contact, and the strain rate at $(R-\Delta)$ is

$$
\dot{\gamma}=\left.\frac{\partial u_{z}(r)}{\partial r}\right|_{r=R-\Delta}=-\frac{\rho F_{e}}{2 \eta_{0}}(R-\Delta) .
$$

By substituting the constitutive Eqs. (9) and (10) in Eq. (8) we can solve for $u_{\text {slab}}$,

$$
-\zeta_{0} u_{\text {slab }}-A \eta_{0} \dot{\gamma}+m F_{e}=0,
$$

giving

$$
u_{\text {slab }}=\frac{\rho F_{e}}{2 \xi_{0}} R .
$$

Finally, by using the slip length definition for this geometry,

$$
L_{s}=-u_{s} /\left.\frac{\partial u_{z}(r)}{\partial r}\right|_{r=R}
$$

we can derive

$$
L_{s}=\frac{\eta_{0}}{\xi_{0}}+\Delta\left[\frac{\Delta}{6 R}-\frac{1}{2}\right]
$$

which means that $\left|L_{s}\right|=\eta_{0} / \xi_{0}$ as $\Delta \rightarrow 0$ in agreement with the Navier slip length definition. The $\Delta$ terms can be neglected for sufficiently wide pores, but when it is comparable to the pore diameter its effect becomes important. For high slip systems, such as the one presently in this study, the effect of the $\Delta$ terms can be neglected even for small diameter CNTs. Here we note, for $\mathrm{R} \approx \Delta$, non-local effects can become important and the friction coefficient includes viscous contributions as well..$^{20,29,30}$

Note that $u_{\text {slab }}$ is equal to the CM velocity of the slab $u_{\mathrm{cm}}$ under the assumption that the fluid density of the slab is constant.

$$
\begin{aligned}
u_{\mathrm{cm}} & =\frac{1}{m} \int_{V} \rho u(r) d V=\frac{\pi L(2 R-\Delta) \rho}{m} \int_{R-\Delta}^{R} u(r) d r \\
& =\frac{1}{\Delta} \int_{R-\Delta}^{R} u(r) d r
\end{aligned}
$$

where the volume of the slab is $V=\pi L \Delta(2 R-\Delta)$ and $\rho=m / V$. 


\section{RESULTS AND DISCUSSION}

We apply the method to determine the friction coefficient for methane confined in CNTs of various diameters and compare our predictions with Poiseuille flow NEMD simulation results. We model the CNTs using the TersoffBrenner (REBO) (Ref. 31) potential and the interactions between fluid-fluid and fluid-solid atoms are modeled using the Lennard-Jones potential with a $1 \mathrm{~nm}$ cutoff distance. The Lennard-Jones interaction potential parameters can be found in Ref. 35. The bulk fluid state point is $(\rho, T)=(387.6 \mathrm{~kg}$ $\left.\mathrm{m}^{-3}, 148.1 \mathrm{~K}\right)$. A Nosé-Hoover thermostat is applied to the wall atoms so that the intrinsic dynamics of the fluid atoms is not affected (compared to if thermostated directly). ${ }^{32} \mathrm{Sim}$ ulations are carried out for 10-40 ns depending on the tube size and external field, using the leap-frog integration algorithm with a time step of $1 \mathrm{fs}$. We have simulated eleven armchair CNTs of diameter ranging from 1.62 to $9.76 \mathrm{~nm}$. For the higher diameter CNTs the curvature effects are expected to be small and both the fluid-tube frictional force and fluid-planar surface (graphene) frictional force are equal and indeed the results show this.

In equilibrium simulations, for each tube we define a cylindrical fluid slab of average width one molecular diameter adjacent to the CNT surface, $\Delta=0.38 \mathrm{~nm}$. After equilibration, we evaluate the slab CM velocity $u_{\text {slab }}(t)$ $=\frac{1}{m} \sum_{i \in \text { slab }} m_{i} v_{i, z}(t)$ and tangential force on the slab due to the wall atoms $F_{z}^{\prime}(t)=\sum_{\substack{i \in \text { salab } \\ j \in \text { wall }}} F_{i j, z}(t)$ in the axial direction. From the above two quantities, we evaluate the slab $\mathrm{CM}$ velocity autocorrelation function $(\mathrm{ACF}) C_{u u}(t)$, and slab velocity-force cross correlation function $(\mathrm{CCF}) C_{u F_{z}^{\prime}}(t)$. In Fig. 2 we plot the slab CM velocity ACF for a short time with the chirality vector ${ }^{33}$ of each CNT indicated on the graph. As the tube diameter is decreased, the slab velocity correlations are found to persist for a longer time, indicating that the fluid dynamics become more correlated. The two correlation functions are then Laplace transformed and the friction coefficient is found using the parameters of the Maxwellian fit and the surface area of the slab. ${ }^{24}$ We find the slip length for each CNT by dividing the shear viscosity of bulk methane with

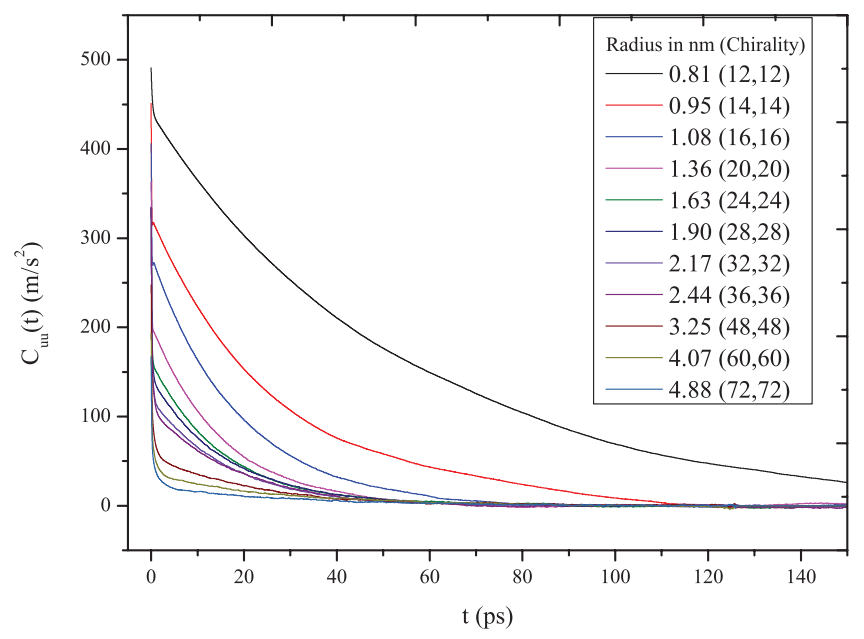

FIG. 2. Slab CM velocity ACF for a short time. The armchair chirality vector $^{33}$ and the radius of each CNT in $\mathrm{nm}$ are indicated on the plot.

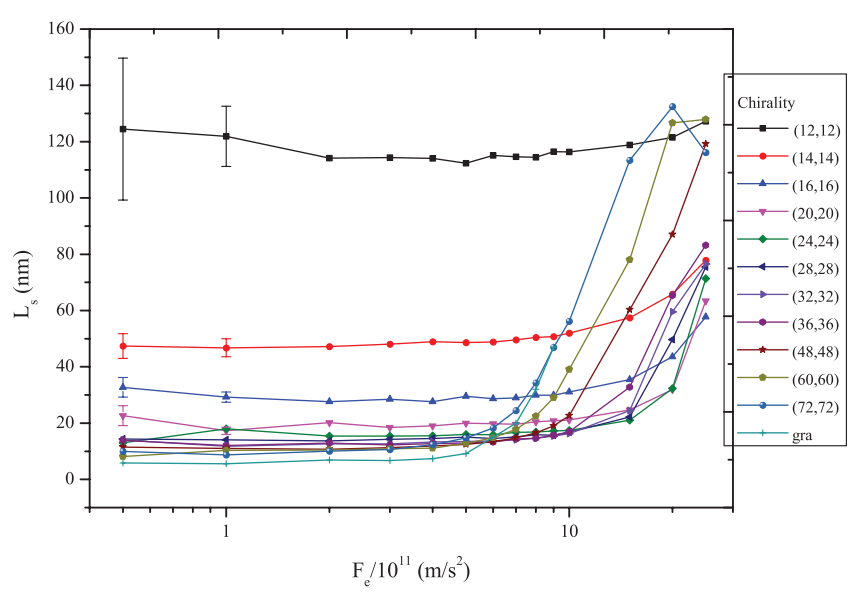

FIG. 3. The slip length as a function of external field for the 11 CNTs studied using NEMD simulations. Also, included the slip length on a planar graphene surface (gra). ${ }^{35}$

the corresponding friction coefficient of the tube. ${ }^{15}$ We shall comment on this later.

In non-equilibrium simulations, Poiseuille flow is generated by applying a range of external fields. We fit the streaming velocities to a quadratic equation $u_{z}(r)=a r^{2}+b$. We constrain the fit such that the parameters satisfy the shear viscosity of bulk methane. Due to the high slip and small external fields, constraining the fit is necessary. ${ }^{34}$ From these fits we find the slip length using Eq. (14). In Fig. 3 we plot the slip length as a function of external field for all the CNTs simulated. The plot also includes the slip length on a planar graphene surface. ${ }^{35}$ As expected, the slip length remains constant for low external fields and increases at high fields. ${ }^{23,35}$ The increase in slip length with field is small in smaller diameter CNTs, which already have a high slip length in the linear regime, whereas in the larger diameter CNTs, the slip length increased rapidly with the external field as compared to smaller diameter CNTs. Hence, the same external field may not result in the linear regime slip length for the same fluid and solid in two different diameter CNTs. In our previous studies we have shown, for the same fluid, the same external field may not give the limiting slip length confined in two different solid material walls. ${ }^{24,35}$ Therefore, care should be taken when interpreting the NEMD results for the slip length of a fluid in different diameter CNTs, which are computed only at a single external field as these NEMD fields are orders of magnitude higher than the experimental fields and hence the slip length may not correspond to the limiting experimental slip length. ${ }^{36}$ The slip length is maximum in the smallest diameter CNT where the curvature effects are large, and as the tube size increases, the slip length decreases and approaches a constant value which is equal to the slip length on a planar graphene surface, as anticipated. This result is in qualitative agreement with Refs. 13, 15, 16, but disagree with the findings in Refs. 11, 12, 17.

Both the equilibrium molecular dynamics (EMD) predicted slip length and the limiting NEMD slip length are plotted as a function of CNT diameter in Fig. 4. As can be seen, our friction method slip length predictions are in good agreement with the direct NEMD data in the linear regime, where 


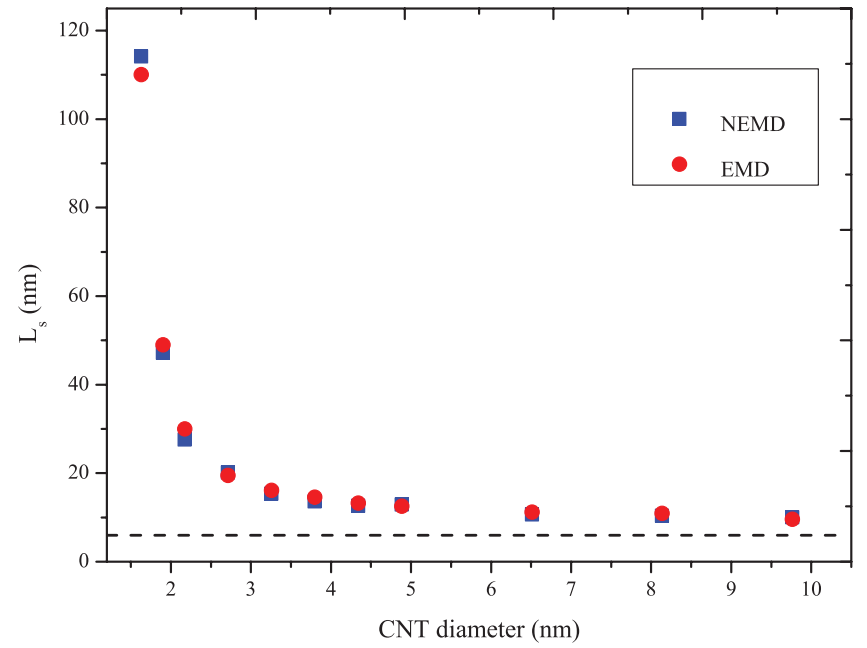

FIG. 4. The slip length as a function of CNT diameter using both EMD and NEMD methods. The dashed line is the slip length on a planar graphene surface. $^{35}$

the slip length is constant and fluid mean velocity increases linearly with the applied pressure gradient. This slip length is valid at experimentally accessible pressure gradients, where the fluid velocity is of the order of $0.01 \mathrm{~m} / \mathrm{s}$. For tube diameters greater than $2 \mathrm{~nm}$, our EMD slip length predictions are within $5 \%$ of the direct NEMD estimates. For a discussion on the superiority of the EMD method over NEMD methods in predicting slip, refer to Refs. 34, 35.

We now comment on the difficulties in predicting the fluid transport properties in very narrowly confined fluids. When fluids are very highly confined (below 3-4 nm or approximately 10 molecular diameters) both the viscosity and the slip length are not well defined. The shear viscosity is defined for a homogeneous fluid based on a continuum hypothesis. ${ }^{1,2}$ Surprisingly even down to roughly $3-4 \mathrm{~nm}$ channel widths confinement, the fluid shear viscosity does not show significant variation. ${ }^{29}$ But below these channel widths, the fluid becomes highly inhomogeneous and fluid density oscillations exist across the whole channel leaving the uniform

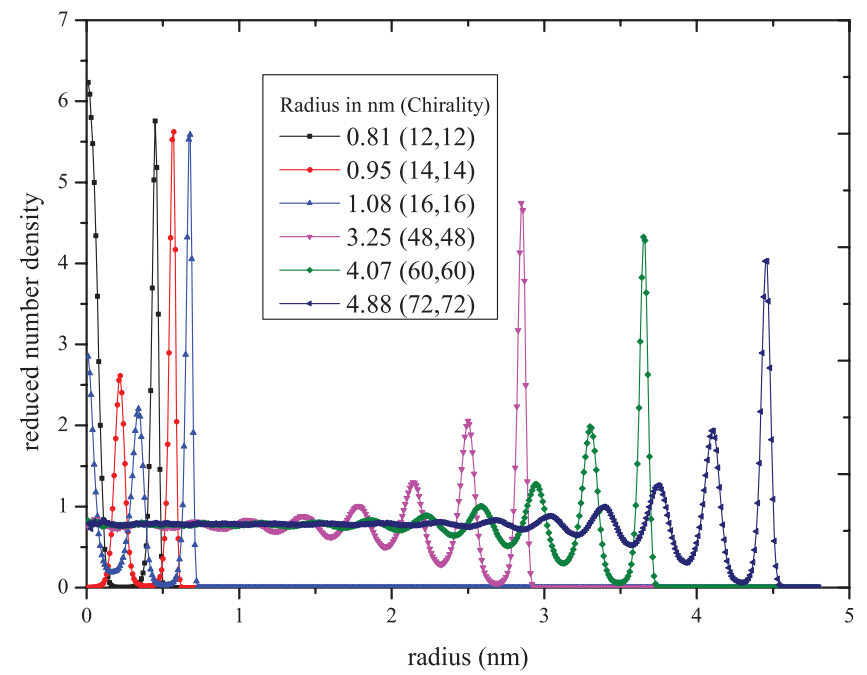

FIG. 5. Density of the fluid in the radial direction for the 3 smallest and 3 widest CNTs simulated. Bulk fluid reduced number density is 0.8 . continuum hypothesis and hence the classical definition of the viscosity invalid. In Fig. 5 we plot the density of the fluid along the radial direction, for the 3 smallest and 3 widest diameter CNTs we have simulated. In the wider diameter CNTs, the density oscillations can be seen near the wall and in the middle of the channel bulk homogeneous fluid can be seen. In the small diameter tubes, we can see inhomogeneity and density oscillations throughout the tube, for which the hypothesis of a uniform continuum is clearly invalid. In these small diameter tubes, the streaming velocity profile need not be quadratic. In simulation studies of water confined in CNTs researchers have found a large variation (increasing, decreasing, non-monotonic) in the behaviour of the effective shear viscosity with the tube diameter. ${ }^{13,15,16,37-40}$ In experimental studies with slit pores of few molecular widths also, the shear behaviour of water is not yet clearly resolved. ${ }^{41-44}$ The discrepancy could be due to the breakdown of existing methods of defining and measuring viscosity for such tightly confined fluids, where non-local effects occur and a viscosity kernel must be considered, ${ }^{29,30}$ which is beyond the scope of this paper. Both the EMD and NEMD slip lengths are proportional to the shear viscosity of the fluid. So the effect of a change in the viscosity is the same for both methods, hence, with a tube diameter dependent effective viscosity also, our friction method results will agree with direct NEMD simulation results.

The slip length definition assumes a quadratic streaming velocity profile for the fluid across the channel which is also based on the constant density continuum hypothesis. ${ }^{1,2,34}$ As this hypothesis is not valid for such narrowly confined fluids the velocity profile may not be quadratic, as has been shown in the literature. ${ }^{45,46}$ Therefore, the slip length definition assuming a quadratic profile for Poiseuille flow may not be valid at this confinement level. Due to the high slip and very small velocity difference of the fluid from centre to the wall we are unable to resolve such a non-quadratic velocity profile even if it exists. ${ }^{34}$

It is generally stated that both the friction coefficient and the slip length of a fluid-solid are intrinsic properties and independent of channel width for a planar boundary. ${ }^{18-20,24,47}$ This is true for fluids confined at the macroscale down to a few nanometers (3-4) channel width. Below these channel widths, they may depend on the channel width due to the above mentioned confinement effects. The uncertainty in defining the available tube diameter also makes a significant difference in the slip length/flow enhancement for small diameter tubes. ${ }^{34}$ There is therefore plenty of room for future research in understanding very narrowly confined fluid behaviour.

\section{CONCLUSION}

In summary, we have presented a method to predict the hydrodynamic BC for fluids confined in cylindrical nanotubes based on the fluid-solid intrinsic interfacial friction coefficient proposed by Navier. The method predicts the non-equilibrium slip phenomenon from equilibrium MD calculations. We have applied the method to methane flow in various diameter CNTs and found good agreement with the direct NEMD results. The slip length is found to decrease monotonically as the tube diameter increases and approaches a constant value, which is 
equal to the slip length on a planar graphene surface. Hence the flow enhancement increases as the diameter of the tube is decreased. Smaller diameter CNTs can be both highly selective and highly permeable and could be used in potential membrane science applications such as nanofiltration. The proposed method is robust, general, and can be used to predict the slip for cylindrical nanofluidic systems.

\section{ACKNOWLEDGMENTS}

This project was supported by the Victorian Partnership for Advanced Computing HPC Facility and Support Services and an award under the Merit Allocation Scheme on the NCI National Facility at the ANU. J. S. Hansen wishes to acknowledge Lundbeckfonden for supporting this work as a part of Grant No. R49-A5634.

${ }^{1}$ H. Lamb, Hydrodynamics (Cambridge University Press, New York, 1993).

${ }^{2}$ G. K. Batchelor, An Introduction to Fluid Dynamics (Cambridge University Press, New York, 2000).

${ }^{3}$ W. Sparreboom, A. v. d. Berg, and J. C. T. Eijkel, New J. Phys. 12, 015004 (2010).

${ }^{4}$ C. Neto, D. R. Evans, E. Bonaccurso, H.-J. Butt, and V. S. J. Craig, Rep. Prog. Phys. 68, 2859 (2005).

${ }^{5}$ G. M. Whitesides, Nature (London) 442368 (2006).

${ }^{6}$ M. Majumder, N. Chopra, R. Andrews, and B. J. Hinds, Nature (London) 438, 44 (2005)

${ }^{7}$ J. K. Holt, H. G. Park, Y. Wang, M. Stadermann, A. B. Artyukhin, C. P. Grigoropoulos, A. Noy, and O. Bakajin, Science 312, 1034 (2006).

${ }^{8} \mathrm{M}$. Whitby and N. Quirke, Nat. Nanotechnol. 2, 87 (2007).

${ }^{9}$ M. Whitby, L. Cagnon, M. Thanou, and N. Quirke, Nano Lett. 8, 2632 (2008).

${ }^{10}$ X. Qin, Q. Yuan, Y. Zhao, S. Xie, and Z. Liu, Nano Lett. 11, 2173 (2011).

${ }^{11}$ E. M. Kotsalis, J. H. Walther, and P. Koumoutsakos, Int. J. Multiphase Flow 30, 995 (2004)

${ }^{12}$ I. Hanasaki and A. Nakatani, J. Chem. Phys. 124, 144708 (2006).

${ }^{13}$ J. A. Thomas and A. J. H. McGaughey, Nano Lett. 8, 2788 (2008).

${ }^{14}$ X. Chen, G. Cao, A. Han, V. K. Punyamurtula, L. Liu, P. J. Culligan, T. Kim, and Y. Qiao, Nano Lett. 8, 2988 (2008).

${ }^{15}$ K. Falk, F. Sedlmeier, L. Joly, R. R. Netz, and L. Bocquet, Nano Lett. 10, 4067 (2010)
${ }^{16}$ J. S. Babu and S. P. Sathian, J. Chem. Phys. 134, 194509 (2011).

${ }^{17}$ V. P. Sokhan, D. Nicholson, and N. Quirke, J. Chem. Phys. 117, 8531 (2002).

${ }^{18}$ L. Bocquet and J.-L. Barrat, Phys. Rev. E 49, 3079 (1994).

${ }^{19}$ V. P. Sokhan and N. Quirke, Phys. Rev. E 78, 015301R (2008).

${ }^{20}$ J. Petravic and P. Harrowell, J. Chem. Phys. 127, 174706 (2007); 128, 209901 (2008) (Erratum).

${ }^{21}$ A. E. Kobryn and A. Kovalenko, J. Chem. Phys. 129, 134701 (2008).

${ }^{22}$ H. Brenner and V. Ganesan, Phys. Rev. E 61, 6879 (2000); 62, 7547 (2000) (Erratum).

${ }^{23}$ P. A. Thompson and M. O. Robbins, Nature (London) 389, 360 (1997).

${ }^{24}$ J. S. Hansen, B. D. Todd, and P. J. Daivis, Phys. Rev. E 84, 016313 (2011).

${ }^{25}$ C. Denniston and M. O. Robbins, J. Chem. Phys. 125, 214102 (2006).

${ }^{26}$ A. Niavarani and N. V. Priezjev, Phys. Rev. E 77, 041606 (2008).

${ }^{27}$ N. V. Priezjev, Phys. Rev. E 82, 051603 (2010).

${ }^{28}$ C. L. M. H. Navier, Mem. Acad. Sci. Inst. Fr. 6, 389 (1823)

${ }^{29}$ P. J. Cadusch, B. D. Todd, J. Zhang, and P. J. Daivis, J. Phys. A: Math. Theor. 41, 035501 (2008).

${ }^{30}$ B. D. Todd, J. S. Hansen, and P. J. Daivis, Phys. Rev. Lett. 100, 195901 (2008)

${ }^{31}$ D. W. Brenner, O. A. Shenderova, J. A. Harrison, S. J. Stuart, B. Ni, and S. B. Sinnott, J. Phys.: Condens. Matter 14, 783 (2002).

${ }^{32}$ S. Bernardi, B. D. Todd, and D. J. Searles, J. Chem. Phys. 132, 244706 (2010).

${ }^{33}$ For a given $(\mathrm{m}, \mathrm{n})$ chirality $\mathrm{CNT}$, the radius is equal to $0.142 \times \sqrt{(3)}$

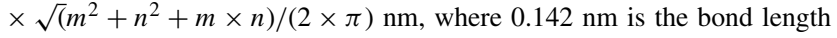
between two carbon atoms.

${ }^{34}$ S. K. Kannam, B. D. Todd, J. S. Hansen, and P. J. Daivis, J. Chem. Phys. 136, 024705 (2012).

${ }^{35}$ S. K. Kannam, B. D. Todd, J. S. Hansen, and P. J. Daivis, J. Chem. Phys. 135, 144701 (2011).

${ }^{36}$ E. Lauga, M. P. Brenner, and H. A. Stone, Experimental Fluid Dynamics (Springer, New York, 2007).

${ }^{37}$ H. Ye, H. Zhang, Z. Zhang, and Y. Zheng, Nanoscale Res. Lett. 6, 87 (2011).

${ }^{38}$ Y. Liu and Q. Wang, Phys. Rev. B. 72, 085420 (2005).

${ }^{39}$ Y. Liu, Q. Wang, T. Wu, and L. Zhang, J. Chem. Phys. 123, 234701 (2005).

${ }^{40}$ H. Zhang, H. Ye, Y. Zheng, and Z. Zhang, Microfluid. Nanofluid. 10, 403 (2011).

${ }^{41}$ L. Bocquet and E. Charlaix, Chem. Soc. Rev. 39, 1073 (2010)

${ }^{42}$ U. Raviv, P. Laurat, and J. Klein, Nature (London) 413, 51 (2001).

${ }^{43}$ Y. Leng and P. T. Cummings, J. Chem. Phys. 125, 104701 (2006).

${ }^{44}$ Y. Zhu and S. Granick, Phys. Rev. Lett. 87, 096104 (2001).

${ }^{45}$ K. P. Travis, B. D. Todd, and D. J. Evans, Phys. Rev. E 55, 4288 (1997).

${ }^{46}$ K. P. Travis and K. E. Gubbins, J. Chem. Phys. 112, 1984 (2000).

${ }^{47}$ M. Cieplak, J. Koplik, and J. R. Banavar, Phys. Rev. Lett. 86, 803 (2001) 